\title{
Ebola Virus Hemorrhagic Fever: A Simulation-Based Clinical Education Experience Designed for Senior Undergraduate Nursing Students
}

\author{
April Manuel $^{1}$, Sandra MacDonald ${ }^{1}$, Sabrina Alani ${ }^{2}$, Donna Moralejo ${ }^{1}$, Adam Dubrowski ${ }^{3}$ \\ 1. School of Nursing, Memorial University of Newfoundland 2. Emergency Medicine, Memorial University \\ of Newfoundland 3. Emergency Medicine, Pediatrics, Memorial University of Newfoundland
}

$\square$ Corresponding author: Adam Dubrowski, adam.dubrowski@gmail.com

Disclosures can be found in Additional Information at the end of the article

\section{Abstract}

In light of the recent Ebola virus disease outbreak, the nursing faculty at Memorial University of Newfoundland, School of Nursing recognized the need to reassess and reinforce undergraduate nursing students' knowledge and skills related to infection prevention and control precautions and the use of personal protective equipment (PPE). Senior nursing students may have a very limited role in the identification of an Ebola case, depending on clinical placement settings, but teaching them about the expanded precautions used in the care of Ebola cases can serve to reinforce understanding of principles, stimulate interest in infection control, and enhance technical skills that are transferable to other patients with infections.

A simulation-based clinical education experience designed for senior students in their final year of studies in the Bachelor of Nursing (Collaborative) Program was developed using a stepwise approach and following the deteriorating patient scenario (DPS) method. The simulation has four implementation frames that are linked to the four key learning objectives, which include the following: (1) recognize Ebola virus disease compatible symptoms, (2) implement the guidelines for expanded isolation precautions to prevent the transmission of the Ebola virus, (3) demonstrate the proper sequence for donning and doffing personal protective equipment (PPE), and (4) remove risk for exposure to the Ebola virus through decontamination. The simulation experience concludes with a video-based debriefing that followed a modified rapid cycle deliberate practice method.

Received 11/03/2014

Review began 11/04/2014

Review ended 11/13/2014

Published 11/17/2014

C) Copyright 2014

Manuel et al. This is an open access article distributed under the terms of the Creative Commons Attribution License CC-BY 3.0., which permits unrestricted use, distribution, and reproduction in any medium, provided the original author and source are credited.
Categories: Emergency Medicine, Medical Simulation, Infectious Disease Keywords: infectious diseases, emergency medicine, simulation based medical education, ebolavirus, nursing education

\section{Introduction}

The current Ebola outbreak ravaging Guinea, Liberia, and Sierra Leone was first detected in March of 2014 and has since spread to other West African regions, with isolated cases in countries such as Spain and the United States. This is the worst outbreak of the Ebola virus in recorded history [1-2]. The Ebola virus is one of the Filoviridae families of viral hemorrhagic fevers [3]. Since the original description of the Zaire Ebolavirus (ZEBOV) in 1976, five different viral subtypes of Ebola have been identified [4]. The causative agent of the most recent outbreak, which occurred initially in Guinea, has been identified as an outlier strain of the ZEBOV and is the most virulent subtype of the Ebola virus [5]. The most common cause of 
transmission of the Ebola virus is through exposure to the bodily fluids of an infected individual, to the mucosal tissues, or abrasions in the skin of an uninfected individual [6]. The incubation period is between 2 and 21 days, with an average incubation period of 7-10 days, and as of yet, there is no vaccine; treatment is supportive [6].

A major concern with the diagnosis of Ebola is that the early clinical symptomatology appears non-specific at first, consisting of fever and myalgia, and followed by flu-like and gastrointestinal symptoms [7]. These symptoms further develop as endothelial damage occurs in multiple tissues and organs with hemorrhage, disseminated intravascular coagulation (DIC), shock, and multi-organ failure seen in cases that progress; the mortality rate in the current outbreak is $50 \%[7-8]$.

The amount of virus contained in blood and body fluids increases as symptoms worsen, increasing the risk of transmission [9]. The risk to healthcare workers caring for these patients is significant; thus, strict adherence to infection control guidelines is paramount. The Public Health Agency of Canada (PHAC) and the Centers for Disease Control and Prevention (CDC) have developed infection control guidelines to ensure that healthcare workers are prepared to meet the global health challenge of caring for a patient with Ebola virus disease and containing the transmission of the Ebola virus [10-11]. The appropriate level of personal protective equipment (PPE), comprehensive education on how to put on (i.e., don) and take off (i.e., doff) the PPE, following the proper decontamination protocols, and a commitment to stringent safety precautions can all help to eliminate the risk of transmitting the Ebola virus from an infected patient [10-11]. Insufficient or inadequate supplies of appropriate PPE and a lack of education on infection control guidelines for healthcare workers are factors that have contributed to the increased risk of transmission of the Ebola virus to healthcare workers during this outbreak [12]. While breaches in practice during the provision of care can lead to exposure, the greatest risk of exposure for healthcare workers occurs during the removal or doffing of the contaminated PPE if adherence to the stringent procedure is not met.

Since nurses represent key frontline healthcare workers who will care for patients experiencing Ebola, they need to be able to recognize Ebola virus disease-compatible symptoms, and implement the relevant protocols, including properly donning and doffing of PPE, and reducing the risk of transmission of the virus through appropriate decontamination.

We anticipate that nursing students will not care for a patient with Ebola virus disease in the current outbreak. Senior nursing students may have a very limited role in the identification of a patient with Ebola virus disease, however. Depending on their clinical placement settings, they may have already witnessed activity on some clinical units related to preparation for the care of suspect Ebola cases. Teaching them about the expanded precautions used in the care of Ebola cases can serve to reinforce the understanding of principles, stimulate interest in infection control and enhance technical skills that can be transferred to other patients with known and suspected infections. In addition, they will be better prepared as new graduates to contribute to a supportive practice environment in the care of patients with infections. We, therefore, see the value of involving senior nursing students in an Ebola-related simulation training exercise. Using pedagogically sound simulation-based clinical education strategies, we describe an experience designed to teach senior nursing students how to appropriately manage a patient presenting with Ebola virus disease; the exercise can also be readily adopted for use with nurses as well.

\section{Technical Report}

This technical report describes a simulation-based clinical education experience designed for senior undergraduate nursing students. The learning objectives of the experience include the following: (1) recognize Ebola virus disease compatible symptoms (2) implement the guidelines 
for expanded isolation precautions to prevent the transmission of the Ebola virus, (3) demonstrate the sequence for donning and doffing personal protective equipment (PPE), and (4) remove the risk for exposure to the Ebola virus through decontamination. This simulation activity is based on guidelines from the CDC for PPE use by healthcare workers during the management of patients with Ebola virus disease. These guidelines are the most readily available, although different local protocols may vary slightly by jurisdiction. For example, most areas in Canada will use disinfectant wipes for decontamination of gloves rather than alcoholbased hand rub; the CDC guidelines identify that either can be used. The symptoms identified in the scenario are consistent with the symptoms identified by the Public Health Agency of Canada national case definitions [10-11].

The simulation-based educational experience takes place in a laboratory setting that mimics the emergency triage area in a hospital emergency department. That area consists of an isolation room with a connecting antechamber where students can don and doff the required PPE. This simulation activity uses a standardized "live" patient as the focus of care and a standardized trained observer. The CDC protocol mandates that a trained observer supervises the donning and doffing procedure, assisting as needed, using a checklist to ensure all steps are completed in the correct sequence.

Prior to the simulation exercise, a case scenario consisting of four implementation frames was designed (Figure 1). The frame design followed a stepwise, simulation-based clinical education experience developed to follow the deteriorating patient scenario (DPS) method [13-15]. In line with the DPS method, each simulation frame has a set of expected actions, and if the actions are correct, the scenario progresses to the next frame or sub-frame. In the event that the student has difficulty with demonstrating the expected actions, the instructor will offer students progressively more revealing and informative "hints" as to the proper course of action. Once students recognize and display the proper actions, the scenario progresses to the next frame.

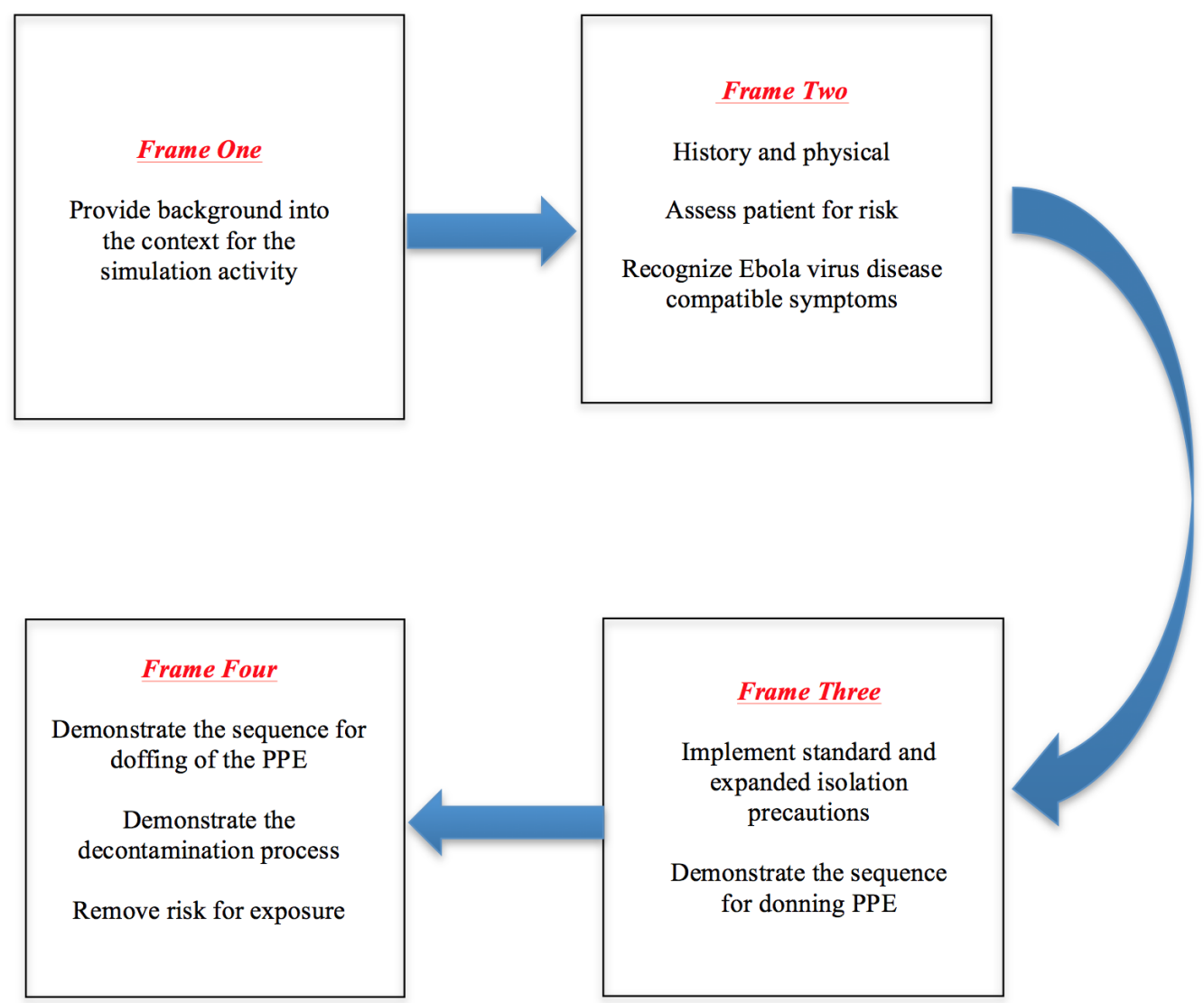




\section{Frame one}

To Provide Background into the Context of the Simulation Activity

Learner Characteristics:

Senior nursing students attend the simulation prepared to demonstrate hand hygiene, use of PPE (e.g., donning gloves and the N95 respirator) and implementation of droplet and contact precautions. The following information regarding the student nurses' role in the scenario is provided in a written format to the student as follows:

"You are an emergency room registered nurse working in the local hospital. You have been assigned the role of triaging patients. Your role is to triage patients according the type and severity of their presenting signs and symptoms. This includes ensuring that the sickest patients are seen first by the emergency room physician. There is an emergency room physician, a Charge Nurse, and four other emergency room nurses on shift. There is also an Infection Control Team in place for patients presenting with the Ebola virus disease.

Patient Characteristics:

The following information regarding the patient in the scenario is provided in a written format to the student:

"Susan is a 34-year-old biology graduate student who has been conducting research in Sierra Leone. She just arrived back in Canada six days ago. She comes to the Emergency Department because she feels unwell and is complaining of a severe headache."

\section{Frame two}

Recognize the Ebola Virus Disease-Compatible Symptoms

Nursing History and Physical Assessment:

The patient is a 34-year-old biology graduate student from the province of Newfoundland. She recently traveled to Sierra Leone to complete a research project on the effectiveness of bat bioacoustics monitoring. Her only past medical history is a congenital heart defect, which is asymptomatic. 


\section{Cureus}

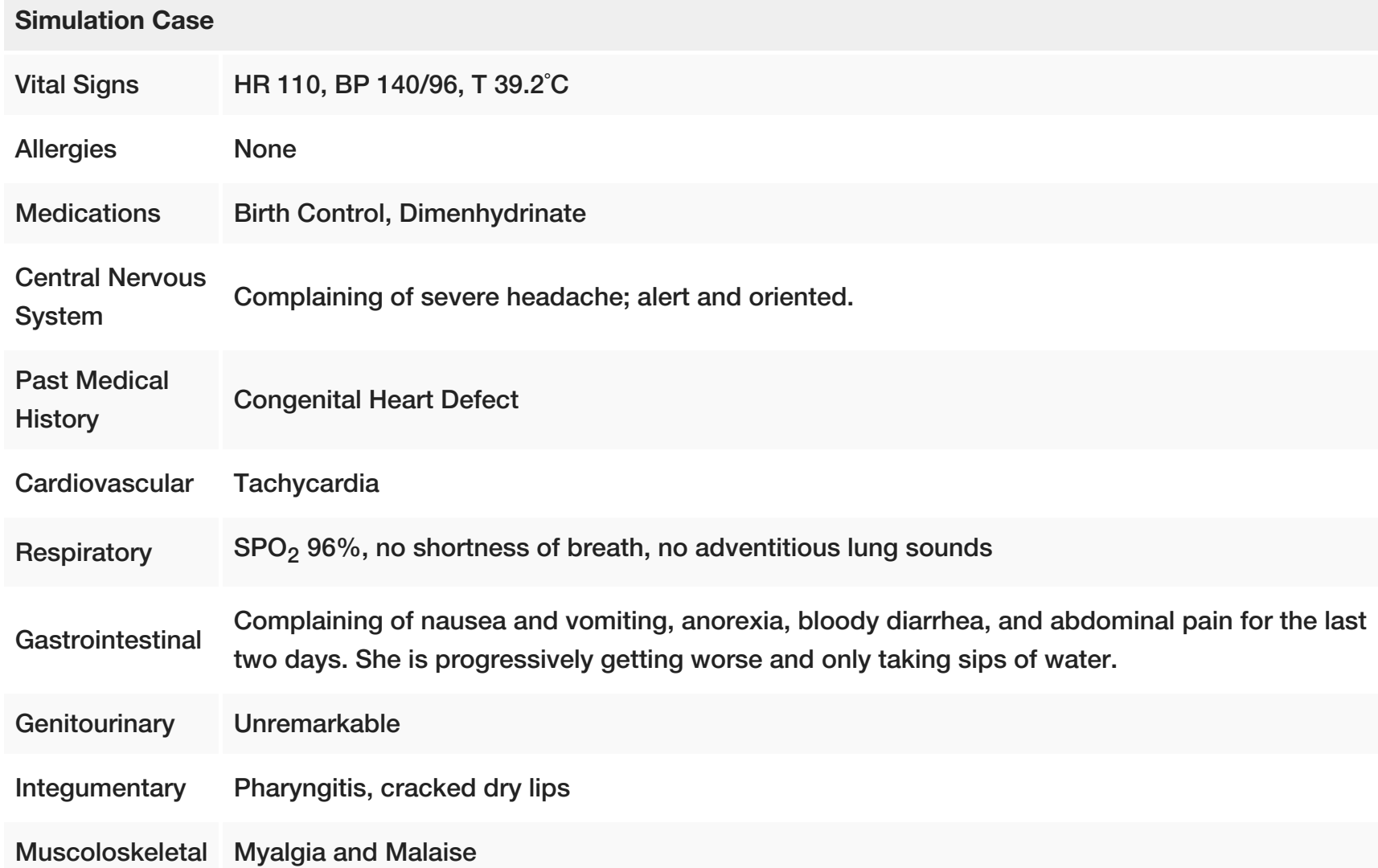

\section{TABLE 1: Physical Assessment Scenario}

Heart Rate (HR) Blood Pressure (BP) Temperature (T)

Expected Actions:

Complete a nursing history and physical

Assess for the Ebola virus disease-compatible symptoms, including temperature $>38.6$ and at least one of the following symptoms:

-Severe headache, erythematous maculopapular rash on the trunk, nausea, vomiting, pharyngitis, bloody diarrhea, myalgia, malaise, abdominal pain, and/or unexplained hemorrhage.

Question the patient for at least one of the following epidemiological risk factors within the past 21 days:

-Resided in or traveled to a country with active Ebola, and/or

-Direct unprotected contact with bats in an Ebola-affected country.

Recognize the patient as a potential Ebola case and explain actions to the patient. 


\section{Cureus}

Notify the charge nurse or the physician that this is a potential Ebola case.

Verbalize the need to implement expanded isolation precautions for Ebola and to alert the Infection Control Team.

\section{HINTS}

Ask student to articulate key concerns for this patient.

Ask the student what system is the focus of attention: Gastrointestinal (GI).

Prompt student to reflect on common causes of GI disturbances.

Ask what would be the concern if temperature $>38.6 \mathrm{C}$, and patient recently traveled to a high-risk country.

Ask student to think about recent travel advisories in the media.

Ask the student to articulate the difference between standard and expanded isolated precautions.

Show student Ebola video.

\section{TABLE 2: Frame 2 Hints}

\section{Frame three}

Part A: Implement the Standard and Expanded Isolation Precautions for Ebola

Expected Actions:

Initiate guidelines for standard and expanded isolation precautions for the transmission of Ebola as per CDC and PHAC guidelines.

Refer to institution's infection prevention control guidelines and policies for the care of a patient with Ebola virus disease.

Put a mask on the patient and brings her to the designated room with anteroom and dedicated washroom facilities.

Keep the door to the patient's room closed.

Place contact and droplet isolation sign on the door.

Explain to the patient the rationale for expanded isolation precautions.

Using the checklist provided, review the PPE stocked in the anteroom and identified if/what additional PPE needs to be obtained.

Identify the anticipated dedicated medical equipment needed to care for the patient (e.g., hands-free garbage, impervious waste-holding bags, no-touch receptacle linen container, bedpan, thermometer, sphygmomanometer, emesis basin, stethoscope etc.). 


\section{Cureus}

Prepare logbook for those entering and exiting isolation room.

\section{HINTS}

What types of precautions are needed in this case?

What type of room is needed to implement the isolation precautions required in this case?

What members of the team would be important to coordinate the care of this woman?

What PPE equipment do you need to care for an Ebola patient?

Ask are there any special considerations you need to consider when preparing the isolation room?

Consider the removal of infectious material from the isolation room, what equipment would you need?

\section{TABLE 3: Frame 3 (Part A) Hints}

Part B: Demonstrate the Proper Sequence for Donning the PPE

Expected Actions:

Select the proper PPE that is recommended by the CDC guidelines, assisted by the trained observer and the checklist provided. The PPE selected should be the appropriate size and material.

(Note: All items are single use (disposable), and fluid resistant or fluid impermeable)

-Two sets of examination gloves with extended cuffs.

-Waterproof boots or washable shoes.

-Waterproof boot covers that go to at least mid-calf, or leg covers.

-A gown that extends to at least mid-calf or coveralls.

-N95 respirator, full-face shield, and a hood that extends to shoulders.

-An apron that covers the torso to the level of the mid-calf (and that covers the top of the boots or boot covers).

Clarify with the trained observer the procedure for following the checklist and obtaining assistance with donning PPE.

Demonstrate correct sequence for donning the PPE including the following:

-Remove personal clothing and items and change into scrubs.

-Remove any personal items (e.g. jewelry) and ensures the hair is away from the face. 


\section{Cureus}

-Inspect PPE prior to donning for defects.

-Perform hand hygiene.

-Put on inner gloves.

-Put on shoe covers.

-Put on gown or coveralls, with assistance for tying if necessary.

-Make sure cuffs of inner gloves are tucked under the gown sleeves.

-Put on an N95 respirator; complete a user seal check.

-Put on a hood.

-Put on an outer apron.

-Put on outer gloves; ensure outer glove cuffs are over gown sleeves.

-Put on face shield over the surgical hood and N95 respirator.

-Verify with trained observer and ensure no skin is exposed.

-Disinfect outer gloves with an alcohol-based hand rub (ABHR) or disinfectant wipe and dry before entering room [16].

Ensure entry into the patient's room is documented in the logbook.

\section{HINTS}

Prompt student to articulate the rationale for PPE with the Ebola virus.

Inform the student that a trained monitor is available to help.

Ask the student what documentation is important prior to entering the room.

Ask the student to reflect on any potential breaks in the procedure that could lead to exposure to the virus.

\section{TABLE 4: Frame 3 (Part B) Hints}

\section{Frame four}

Demonstrate the proper removal of the PPE and the decontamination process

Remove PPE without self-contamination or contamination of the environment.

Provide the rationale as to the sequence of PPE removal. 
Clarify with the trained observer the procedure for following the checklist and obtaining assistance with doffing PPE.

With guidance and assistance from the trained observer, demonstrate the proper sequence for removing PPE:

-Inspect PPE for any tears, contamination, or cuts.

-If contamination is visible on any PPE, disinfect with ABHR or disinfectant wipes.

-Disinfect outer gloves with ABHR or disinfectant wipes.

-Remove apron; roll from inside to outside.

-Inspect PPE again as above.

-Disinfect outer gloves with ABHR or disinfectant wipes.

-Remove shoe covers while sitting down and discard.

-Disinfect and remove outer gloves.

-Inspect and disinfect inner gloves.

-Remove inner gloves, perform hand hygiene, and don a clean pair of gloves.

-Remove face shield.

-Disinfect inner gloves.

-Remove surgical hood and discard.

-Disinfect inner gloves.

-Remove gown:

Unfasten ties and slip hands under the gown at the neck and shoulder and peel gown away from neck and shoulder (monitor may help to unfasten ties and pass ties to student)

Pull away from the body, rolling from inside to out and touching only the inside of the gown. Turn contaminated outside toward the inside. Only the clean part of the gown should be visible.

-Disinfect and change inner gloves. Don a clean pair of gloves.

-Remove N95 respirator.

-Disinfect inner gloves.

-Disinfect washable shoes. 


\section{Cureus}

-Disinfect inner gloves and remove.

-Perform hand hygiene.

-Final inspection [16].

Respond appropriately to breaks in the protocol or potential breaches (e.g., additional decontamination, changing gloves), identifying the potential sources of contamination and provides rationales.

PPE is disposed of properly and placed in a leak proof infectious waste container.

Ensure entry into the patient's room is documented in the logbook.

\section{HINTS}

Prompt the student to ask the monitor to assist with the removal of PPE.

Guide the student through the process of taking off the equipment: What is the first piece of PPE to be removed and why?

Ask the student what is the final step once all PPE is removed (final inspection).

Prompt the student as to how they would document time spent in the isolation room.

Ask the student to reflect on any potential breaks in the protocol.

TABLE 5: Frame 4 Hints

Discussion

\section{Debriefing}

Feedback and debriefing were accomplished in two ways. First, during the simulation and because the skills involved are very procedural, we have selected to follow the modified rapid cycle deliberate practice method as outlined by Hunt, et al. [17]. When errors or unwanted actions were spotted, the educator would stop the student and provide them with feedback in the form of progressively more informative hints. The student would be then asked to repeat the frame that contained the error or unwanted action while incorporating the hints and feedback. In additions, the entire encounter with the Ebola-simulated patient is video recorded and used for debriefing. The student and the educator review the video recording together, and the educator pauses the video at any time that a breach occurred or potentially could have occurred or where there was difficulty with any aspect. The educator would follow the "frame discovery" approach [18] and ask the student to describe the reason for taking a particular action or to explain the source of the breach or difficulty. Subsequently, the educator and student would discuss appropriate actions and rationales. In addition, to assess understanding of enhanced precautions for Ebola virus disease compared to contact/droplet precautions for influenza, the student would be asked to identify what would have happened if the patient had influenza and not Ebola virus disease. 


\section{Conclusions}

This technical report described a simulated clinical education experience designed for senior undergraduate nursing students. The learning objectives of the simulated teaching experience included: (1) recognition of the clinical presentation of an individual with Ebola virus disease, (2) implementation of the guidelines for isolation precautions to prevent the transmission of the Ebola virus, (3) demonstration of the sequence for donning and doffing personal protective equipment (PPE), and (4) removal of risk from exposure to the Ebola virus.

The simulation was designed based on the DPS method, and a set of required actions and hints were prepared in order to guide the students through the scenario. In addition, a modified, video-based rapid cycle deliberate practice method for post-simulation debriefing is used, as it is best suited for this type of skill and this level of students.

Although we acknowledge that the protocols for dealing with Ebola virus disease infected patients are changing rapidly, we have adapted the best available protocols at the time of preparation of this technical report. However, the general method of presenting the simulated patient is modular and accommodating to follow any future updates to the protocols.

\section{Additional Information}

\section{Disclosures}

Human subjects: All authors have confirmed that this study did not involve human participants or tissue. Animal subjects: All authors have confirmed that this study did not involve animal subjects or tissue. Conflicts of interest: In compliance with the ICMJE uniform disclosure form, all authors declare the following: Payment/services info: All authors have declared that no financial support was received from any organization for the submitted work. Financial relationships: All authors have declared that they have no financial relationships at present or within the previous three years with any organizations that might have an interest in the submitted work. Other relationships: All authors have declared that there are no other relationships or activities that could appear to have influenced the submitted work.

\section{Acknowledgements}

This project was supported by Tuckamore Simulation Research Collaborative and Emergency Medicine Education Committee, Memorial University of Newfoundland.

\section{References}

1. Butler D: Global Ebola response kicks into gear at last . Nature. 2014, 513:469. 10.1038/513469a

2. Editorial: Ebola: protection of health workers on the front line . Lancet. 2014, 384:470. 10.1016/S0140-6736(14)61319-2

3. Easter A: Ebola: No antiviral compounds are available to treat this hemorrhagic fever . AJN. 2002, 102:49-52. 10.1097/00000446-200212000-00017

4. Gostin LO, Lucey D, Phelan A.: The Ebola epidemic: a global health emergency. JAMA. 2014, 312:1095-1096. 10.1001/jama.2014.1117

5. Baize S, Pannetier D, Oestereich L, et al.: Emergence of Zaire Ebola virus disease in guinea a preliminary report. NEJM. 2014, 371:1418-1425. 10.1056/NEJMoa1404505

6. Ansari AA: Clinical features and pathobiology of Ebolavirus infection. J Autoimmun. 2014, 55:1-9. 10.1016/j.jaut.2014.09.001

7. Fauci AS: Ebola - underscoring the global disparities in health care resources . NEJM. 2014, 371:1084-6. 10.1056/NEJMp1409494

8. Mwanatambwe M, Yamada N, Arai S, Shimizu-Suganuma M, Shichinohe K, Asano G: Ebola hemorrhagic fever (EHF): mechanism of transmission and pathogenicity. J Nippon Med Sch. 
2001, 68:370-375. 10.1272/jnms.68.370

9. Decker BK, Sevransky JE, Barrett K, Davey RT, Chertow DS: Preparing for critical care services to patients with Ebola. Ann Intern Med. 2014, 161:831-2. 10.7326/M14-2141

10. Public Health Care Agency of Canada: Interim Guidance-Ebola Virus Disease: Infection Prevention and Control Measures of Borders, Healthcare Settings and Self-Monitoring at Home. Canada Communicable Disease Report. 2014, 40:15. Accessed: October 24, 2014: www.phac-aspc.gc.ca/id-mi/vhf-fvh/ebola-ipc-pci-eng.php.

11. CDC Tightened Guidance for U.S. Healthcare Workers on Personal Protective Equipment for Ebola. (2014). Accessed: October 24, 2014: http://www.cdc.gov/media/releases/2014/fs1020ebola-personal-protective-equipment.html.

12. Siegel JD, Rhinehart E, Jackson M, Chiarello L, the Healthcare Infection Control Practices Advisory Committee: Guideline for Isolation Precautions: Preventing Transmission of Infectious Agents in Healthcare Settings. Centers for Disease Control and Prevention. 2007, Accessed: October 24, 2014: http://www.cdc.gov/hicpac/2007IP/2007ip_part4.html.

13. Wiseman J, Snell L: The Deteriorating Patient: A realistic but 'low-tech' simulation of emergency decision-making. The Clinical Teacher. 2008, 5:93-95. 10.1111/j.1743498X.2008.00213.x

14. Renouf T, Mitchell K, Hollett S, et al.: Deteriorating Patient Scenario for Simulation-Based Training of Difficult Patient Encounters in the Emergency Room. Cureus. 2014, 6:e192. 10.7759/cureus.192

15. Black H, Renouf T, Parsons M, et al.: Pregnancy and Privacy in an Emergency Department: A Simulated Session. Cureus. 2014, 6: e216. 10.7759/cureus.216

16. Guidance on Personal Protective Equipment To Be Used by Healthcare Workers During Management of Patients with Ebola Virus Disease in U.S. Hospitals, Including Procedures for Putting On (Donning) and Removing (Doffing). (2014). Accessed: October 24, 2014: http://www.cdc.gov/vhf/ebola/healthcare-us/ppe/guidance.html.

17. Hunt EA, Duval-Arnould JM, Nelson-McMillan KL, Bradshaw JH, Diener-West M, Perretta JS, Shilkofski NA: Pediatric resident resuscitation skills improve after "rapid cycle deliberate practice" training. Resuscitation. 2014, 85:945-51. 10.1016/j.resuscitation.2014.02.025

18. Rudolph JW, Simon R, Rivard P, Dufresne RL, Raemer DB: Debriefing with good judgment: combining rigorous feedback with genuine inquiry. Anesthesiol Clin. 2007, 25:361-76. 10.1016/j.anclin.2007.03.007 